DOI https://doi.org/10.30525/978-9934-588-81-5-1.26

\title{
SURGICAL REPAIR OF PECTUS EXCAVATUM - EXPERIENCES WITH NUSS TECHNIQUE IN CHILDREN
}

\author{
Kaminska M. O. \\ PhD, \\ Assistant Professor at the Department of the Pediatric Surgery, \\ Orthopedics and Traumatology \\ Dnipropetrovsk Medical Academy of the Ministry of Health of Ukraine \\ Dnipro, Ukraine
}

Characteristic feature of pectus excavatum (PE) is tendency to progression, which is closely linked with a growth and age of children. Further development of PE leads to the more severe disorders of lungs, to the displacement and rotation of a heart, as a result - to the decompensation of cardiovascular and respiratory systems $[3,2]$.

It is well - known surgical treatment of PE, because conservative treatment is not effective. It was offered more than 80 different variants of surgical procedures and their modifications for PE correction, but there is a high percentage of unsatisfactory results - to $41.7 \%[1,4]$, which indicated about a relevant and unresolved problem in general, and to the necessity of further research in this field.

According to Petersen C. [5], variety of open surgery techniques represented the «golden standard» for remodeling the chest wall of patients with pectus excavatum. This situation changed when a minimally invasive approach was first introduced by D. Nuss. This procedure was originally developed only for children and is based on the fact that the growing chest wall is still flexible. The new technique is gaining acceptance worldwide in the field of pediatric surgery, and older patients who resist open surgery for correction of their pectus excavatum now request the new and less invasive procedure.

Kelly RE Jr. [6] study pectus excavatum indications for surgical treatment, which include two or more of the following: a severe, symptomatic deformity; progression of deformity; paradoxical respiratory chest wall motion; computer tomography scan with a pectus index greater than 3.25; cardiac compression/displacement and/or pulmonary compression; pulmonary function studies showing restrictive disease; mitral valve prolapse, bundle branch block, or other cardiac pathology secondary to compression of the heart; or failed previous repairs. 
Our research aimed to analyze results of the surgical treatment of PE at the children with using minimally invasive method of thoracoplasty.

Our observation and treatment covered 50 children with PE, aged from 4 to 17 years. There were $42(84 \%)$ boys, $8(16 \%)$ girls. Preoperative examination included conduction of the clinical laboratory tests, spirography, electrocardiography, echocardiography, X-ray chest examination in the frontal and lateral projections, computed tomography - by individual indicators. Conducted examination helped to identify ventilation and respiratory disfunctions in the lungs. According to the data of spirography, a lung capacity was decreased, and function of external respiration by restrictive or obstructive type was impaired among $71.43 \%$ of children. At the oldest age group of children was dominated restrictive type of the respiratory failure, primary, because of limiting the mobility of ribs, diaphragm and reducing volume of air. Significant changes were observed in the cardiovascular system on the ECG examination. It was revealed rhythm disturbance in a form of bradycardia and tachycardia at the 28 and $57 \%$ of children. Phenomenon of conduction disorders having $71.43 \%$ of patients in the form of atrioventricular and incomplete blockade in a right bundle of His, overload in the right side of heart. Echocardiography allows to specify morphological changes in the heart, in $52.38 \%$ of cases were observed a mitral valve prolapse. Radiologically was determined degree of deformation of the thorax, using index of Hizhitska. It was diagnosed II stage $18(36 \%)$ and III stage - $32(64 \%)$. A computed tomography was performed in order to determine relative position of mediastinum to the sternocostal complex, and for purpose of assessment the brunch - pulmonary system.

In all cases was conducted a surgical treatment Nuss technique. Results of thoracoplasty by application method of D. Nuss were assessed in 50 patients. For the assessment of clinical efficiency of surgical treatment patients with PE we analyzed results in the early postoperative and remote periods (after removal of the metal constructions).

Results of surgical treatment in the early postoperative period were evaluated according to the intraoperative blood loss, duration of surgery, duration of anesthesia, narcotic analgesics, term of verticalization of the patients, presence of complications.

At the patients, operated by this technique, were not performed hemotransfusions of the red blood cell and blood products. Intraoperative blood loss in the operated patients was varied from 50 to $80 \mathrm{ml}$ during the skin incision and forming a space for conduction a plate and its fixation. Average duration of surgery was ranged from 60 to 110 minutes. Anesthesia was conducted with narcotic analgesics for 1-2 days and then with non- 
narcotic analgesics for 5-7 days. Using of epidural anaesthesia improved at the 5 patients the analgesic effect. Catheter was removed on the $2-3$ day. Majority of children were transferred to a vertical position, which allowed them to start walking on the 2nd day.

Among the complications at the early postoperative period were hemothorax and pneumothorax. Hemothorax was observed in 7 (23.8\%) patients. In the ultrasonography was revealed on average $100 \mathrm{ml}$ of fluid. In $2(9.52 \%)$ cases were performed thoracocentesis as the passive drainage of pleural cavity, in 4 (19.04\%) children was carrying out a pleural puncture. In one patient was developed an exudative plevritis and pericarditis, which were blocked by applying drainage of the pleural cavity and pericardium, a long-term antibiotic therapy.

In the long-term postoperative period at the 3 patients (14.28\%) was appeared seromes in the region of postoperative wound on the right. Their elimination was carried out by excision of the pathologically changed tissues, imposition of the secondary sutures, antibiotic therapy.

To the complications in a long-term period was carried out instability of the implant. In $2(9.52 \%)$ patients was observed shift of the metal construction, caused by its deformation in 1 month and after 1.5 months after surgery. The urgent patients was performed the surgery - it was removed a plate. In one case was conducted the surgery after 8 months without any complications. In the second case, from repeated operations declined, resulting in a relapse of the deformity. In the second case, a patient was not agreeing to the surgery, as the result we found out relapse of the given deformations. This case was related to the unsatisfactory results.

Long-term results of treatment have been traced in a period from 6 months to 4 years after surgery. We evaluated results of the surgery on the following criteria: good (deformation is eliminated completely, anthropometric indices correspond to the age, cosmetic result is satisfied the patient, functional studies do not reveal deviations from the norm); satisfactory (a partial recurrence of the deformation, the index of Hizhitska should not be less than 0.7 , or curvature of the ribs, complaints on cosmetic defects are missing, or minimal, functional parameters are normal); unsatisfactory (complete relapse of the deformations, complaints on the cosmetic defect). At the research group of patients good results were obtained in $43(86 \%)$ cases, satisfactory in $6(12 \%)$ cases, unsatisfactory - in $1(2 \%)$ case.

Therefore, innovative thoracoplasty technique, which we conducted by the method of D. Nuss is the less traumatic, than traditional methods of treatment. It allows us to reduce duration of postoperative staying of patient in the hospital and obtain good cosmetic results. Analysis of postoperative complications and unsatisfactory results of treatment showed, that the giv- 
en method required improvement of a quality of applied metal clamps in order to increase their strength properties, as well as technical support the possibility of their modeling during the operation.

\title{
References:
}

1. Krivchenia D.Yu. et al. Variants of modifications the Nuss operation in a correction of the funnel chest deformation: advantages and disadvantages, Surgery of children's age, 2011, 1: 27-30.

2. Malakhov OA, Zherdev KV, Chelpachenko OB. Orthopedic aspects and features of surgical treatment of the funnel chest deformation in the children and adolescents, Bulletin of traumatology and orthopedics, named by N.N. Priorova, 2011, 3: 3-9.

3. Plyakin VA, Kulik IO, Sarukhanyan OO. Comparative assessment of the operations by Nuss and Ravich for treatment of the funnel chest deformation, Pediatric surgery, 2013, 3: 60-64.

4. D. Nuss, R.E. Kelly Jr., D.P. Croitoru, M.E. Katz. A 10-year review of a minimally invasive technique for the correction of pectus excavatum, J. Pediatr. Surg., 1998, Vol. 33 (4): 545-552.

5. Petersen C. Funnel chest. New aspects since introduction of a minimally invasive surgical technique, Orthopade. 2003 Oct;32(10):916-9.

6. Kelly RE Jr. Pectus excavatum: historical background, clinical picture, preoperative evaluation and criteria for operation, Semin Pediatr Surg. 2008 Aug;17(3):181-93.

DOI https://doi.org/10.30525/978-9934-588-81-5-1.27

\section{INFLUENCE OF ANDROGEN DEFICIENCY ON THE FORMATION OF DISORDERS OF THE CARDIOVASCULAR SYSTEM DURING PUBERTY, TAKING INTO ACCOUNT CHANGES IN OXIDATIVE BALANCE}

\author{
Kashkalda D. A. \\ PhD, Senior Researcher, \\ Leading Researcher at the Laboratory \\ of Age-related Endocrinology and Metabolism \\ Institute for Children and Adolescents Health Care \\ of the National Academy of Medical Sciences of Ukraine
}

